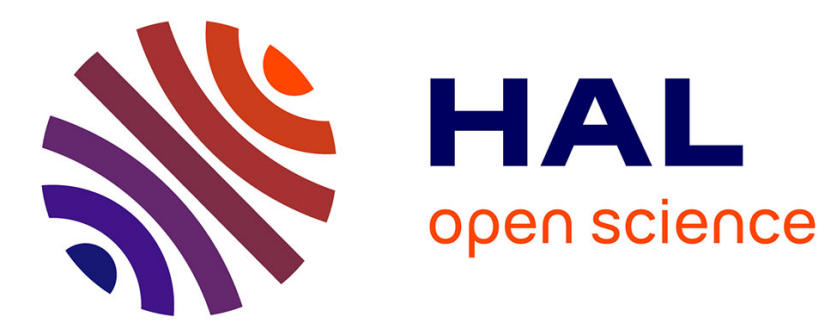

\title{
Statistical Analysis of Bidirectional Reflectance Distribution Functions
}

Carlos J. Zubiaga, Laurent Belcour, Carles Bosch, Adolfo Muñoz, Pascal Barla

\section{To cite this version:}

Carlos J. Zubiaga, Laurent Belcour, Carles Bosch, Adolfo Muñoz, Pascal Barla. Statistical Analysis of Bidirectional Reflectance Distribution Functions . Measuring, Modeling, and Reproducing Material Appearance 2015, Feb 2015, San Francisco, United States. hal-01123655

\section{HAL Id: hal-01123655 \\ https://inria.hal.science/hal-01123655}

Submitted on 11 Mar 2015

HAL is a multi-disciplinary open access archive for the deposit and dissemination of scientific research documents, whether they are published or not. The documents may come from teaching and research institutions in France or abroad, or from public or private research centers.
L'archive ouverte pluridisciplinaire HAL, est destinée au dépôt et à la diffusion de documents scientifiques de niveau recherche, publiés ou non, émanant des établissements d'enseignement et de recherche français ou étrangers, des laboratoires publics ou privés. 


\title{
Statistical Analysis of Bidirectional Reflectance Distribution Functions
}

\author{
Carlos J. Zubiaga ${ }^{a}$, Laurent Belcour $^{a}$, Carles Bosch $^{b}$, Adolfo Muñoz $^{c}$ and Pascal Barla ${ }^{a}$ \\ ${ }^{a}$ Inria - Bordeaux University - IOGS - CNRS (France) \\ ${ }^{b}$ University of Girona - Barcelona Media (Spain) \\ ${ }^{c}$ Universidad de Zaragoza (Spain)
}

\begin{abstract}
Bidirectional Reflectance Distribution Functions (BRDFs) are commonly employed in Computer Graphics and Computer Vision to model opaque materials. On the one hand, a BRDF is a complex 4D function of both light and view directions, which should ensure reciprocity and energy conservation laws. On the other hand, when computing radiance reaching the eye from a surface point, the view direction is held fixed. In this respect, we are only interested in a 2D BRDF slice that acts as a filter on the local environment lighting. The goal of our work is to understand the statistical properties of such a filter as a function of viewing elevation. To this end, we have conducted a study of measured BRDFs where we have computed statistical moments for each viewing angle. We show that some moments are correlated together across dimensions and orders, while some others are close to zero and may safely be discarded. Our study opens the way to novel applications such as moment-based manipulation of measured BRDFs, material estimation and image-based material editing. It also puts empirical and physically-based material models in a new perspective, by revealing their effect as view-dependent filters.
\end{abstract}

Keywords: Bidirectional Reflectance Distribution Functions, Moment-based analysis, Material appearance

\section{INTRODUCTION AND RELATED WORK}

The Bidirectional Reflectance Distribution Function (BRDF) has been introduced by Nicodemus ${ }^{1}$ as a means to characterize the reflectance properties of opaque materials. It is a $4 \mathrm{D}$ function of two directions (e.g., light and view directions), that must be reciprocal (input directions may be swapped without reflectance being changed) and energy-conserving. The BRDF characterizes how much radiance is reflected in all lighting and viewing configurations, and may be considered as a black-box encapsulating light transport at a microscopic scale.

Rendering in Computer Graphics traditionally makes use of BRDFs through the reflected radiance equation, as first introduced by Kajiya: ${ }^{2}$

$$
R\left(\mathbf{p}, \boldsymbol{\omega}_{o}\right)=\int_{\Omega_{\mathbf{n}}} \rho\left(\mathbf{p}, \boldsymbol{\omega}_{o}, \boldsymbol{\omega}_{i}\right) L\left(\mathbf{p}, \boldsymbol{\omega}_{i}\right) \boldsymbol{\omega}_{i} \cdot \mathbf{n} d \boldsymbol{\omega}_{i},
$$

with $R$ the reflected radiance, $\mathbf{p}$ a surface point of interest, $\boldsymbol{\omega}_{o}$ and $\boldsymbol{\omega}_{i}$ the outgoing and ingoing directions, $\mathbf{n}$ the surface normal, $\rho$ the BRDF, $L$ the incoming lighting and $\Omega_{\mathbf{n}}$ the upper hemisphere.

Figure 1 shows renderings of spheres made of five different materials in two different environment illuminations in orthographic view. These images have been obtained by computing the radiance $R$ reflected from every visible surface point toward a pixel in the image. Light transport will be more involved for more complex scenes, but Equation 1 will still remain valid and produce physically-plausible images. Nevertheless, even with the simple scenes of Figure 1, the effect of a particular BRDF in the picture is not, to the best of our knowledge, properly understood. For instance, Hunter has identified six visual properties of gloss in his seminal book on appearance measurement: ${ }^{3}$ specular gloss, sheen, luster, absence-of-bloom, distinctness-of-image and surface-uniformity. He suggests that, except for surface-uniformity, all of these visual properties may be connected to reflectance (i.e., BRDF) properties. There exists standard test methods for measuring some of these properties (such as ASTM D523, D430 or D4039), and recent work has studied how they are related to human vision. ${ }^{4}$ However, we believe that explicit connections between physical and visual properties of materials (independently of any standard or observer) remain to be established. 

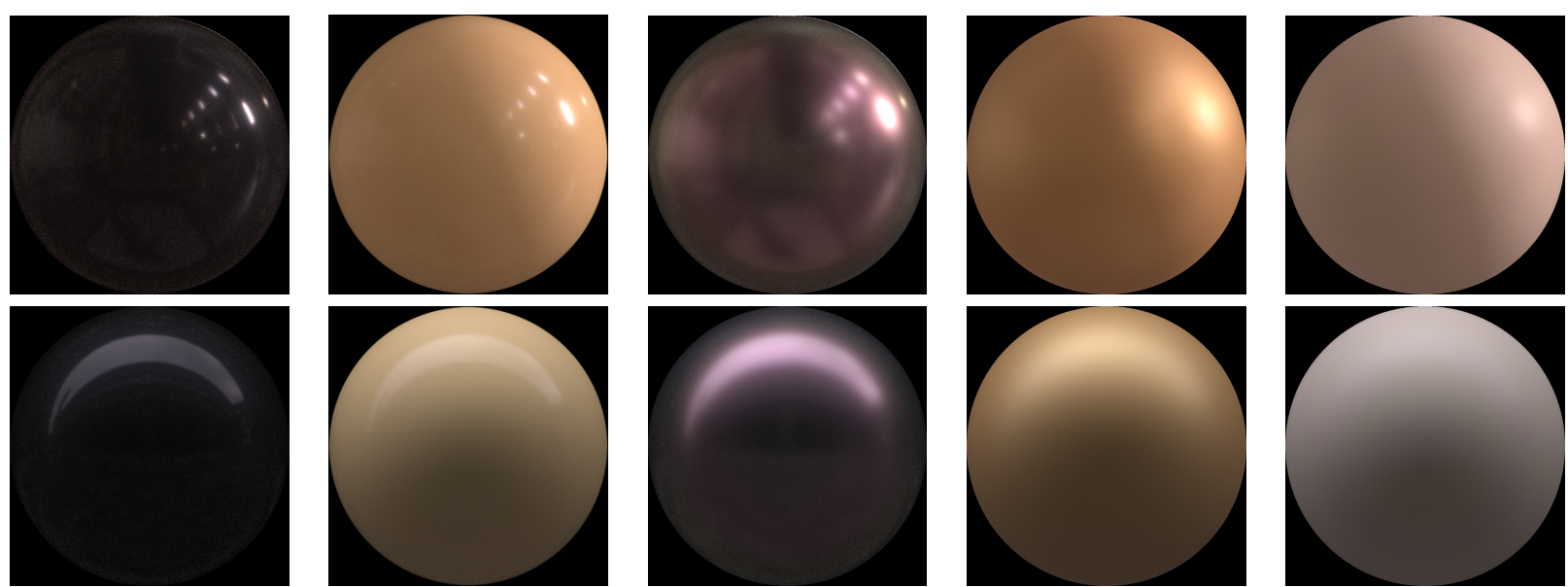

Figure 1. Renderings of different BRDF coming from the MERL database (from left to right: specular-black-phenolic, yellow-phenolic, color-changing-paint2, gold-paint and neoprene-rubber ) under two different environment maps (upper row: galileo; lower row: uffizi). Each BRDF has a different effect on the reflected image of the environment.

The main objective of our work is two-fold: 1) identify relevant BRDF properties that may be observable in a rendered image; and 2) find explicit relationships between properties of BRDFs and reflected radiance.

One way to identify BRDF properties consists in fitting parametric BRDF models to data, and consider parameter values and ranges as relevant properties. Ngan et al. ${ }^{5}$ have conducted such an empirical study, using as input measured materials coming from the MERL database. ${ }^{6}$ This database holds 100 measured BRDFs and displays a wide diversity of material appearances. All BRDFs are isotropic, which means light and view directions may be rotated around the local surface normal with no incurring change in reflectance. The main issue with this approach is that parameters are limited by the choice of models that only span a subset of real-world BRDFs. Recently, it has been reported that statistical moments ${ }^{7}$ may constitute a tool of choice for applications in graphics and vision. The material estimation technique of Ghosh et al. ${ }^{8}$ employs 3D moments (in cartesian space) up to order 2 to recover basic BRDF parameters from a few views and controlled lighting. 1D moments up to order 2 have been used to roughly derive material categories and guide rendering with global illumination. ${ }^{9}$ In this paper, we also rely on statistical moments; however, since our purpose is on the analysis of measured BRDF properties, we study moments up to order 4 for a dense sampling of viewing directions. Moreover, we introduce a new 2D parametrization that stays more faithful to reflectance distributions occurring on a hemisphere.

Recovering BRDF or lighting properties from image properties (i.e., inverse rendering) has been a longstanding goal in Computer Vision. In this paper, we rather take the opposite approach (i.e., forward rendering), as we want to finely understand how specific choices of BRDF or lighting properties influence the final picture. To this end, we turn to rather recent work in Computer Graphics that has conducted such analysis, directly working from Equation 1. A pioneering approach is the work of Ramamoorthi et al. ${ }^{10}$ who make use of Spherical Harmonics to analyze image formation. In particular, they provide limits on observability of BRDF or lighting, situations in which they could hardly be recovered. Durand et al. ${ }^{11}$ rather employed a local spatial Fourier analysis to understand how each light transport event might influence the final image in terms of frequency content. It has been extended to deal with sequences of events occuring along a light path in the 5D covariance tracing technique of Belcour et al. ${ }^{12}$ The locality of analysis is pushed even further in the differential analysis of Ramamoorthi et al., ${ }^{13}$ which provides interesting insights on how surface curvature influences image gradients. The main limitation of these approaches regarding our purpose is that they consider only simple BRDFs: either diffuse, specular, or radially symmetric like the Phong model. ${ }^{14}$ We thus provide a complementary analysis that focuses on the complexity of real-world BRDFs: our approach works locally in Fourier space and provides as a result direct relationships between the moments of the BRDF and reflected radiance.

The remainder of the paper is organized as follows. Section 2 explains how we pre-process BRDF data before computing their moments. In particular, we explain how we separate diffuse and specular components, and 
map hemispherical reflectance distributions to a planar 2D parametrization with minimal distortions. We then compute 2D moments up to order 4 and provide a qualitative analysis that allows us to foresee how such BRDF properties may impact appearance in pictures. Throughout Section 3, we focus on 2D moments up to order 2 to provide a quantitative (albeit approximate) analysis of material appearance. We first express Equation 1 in convolution form which, thanks to a local Fourier analysis, leads to simple expressions that connect BRDF and lighting moments to reflected radiance moments for a fixed viewing direction. We then fit simple analytical functions to moment profiles as a function of viewing elevation, which provides us with more global insights on material appearance. We close our study with a discussion of its limitations in Section 4, where we suggest potential directions for future work.

\section{BRDF MOMENTS}

As is made explicit by Equation 1, a $\operatorname{BRDF} \rho$ is always evaluated at a given surface point $\mathbf{p}$ for a fixed view direction $\boldsymbol{\omega}_{o}$. This restricts a general $4 \mathrm{D}$ BRDF to a 2D BRDF slice, whose statistical properties are the subject of our study. Intuitively, such a slice may be seen as a filter applied to the environment illumination. We suggest that some statistical properties of this filter may be directly observable in images, and thus may constitute building blocks for material appearance.

Our approach is to compute 2D statistical moments of BRDF slices as functions of the viewing direction, up to order 4: energy, mean, co-variance, co-skewness and co-kurtosis. We first provide definitions for the 2D moments onto which we ground our analysis, and show the restrictions they impose on the type of BRDF data we take as input. We then compute moments on a subset of the MERL database; hence we only consider isotropic BRDFs (3D functions ${ }^{6}$ ) with reflectances given in RGB colors. On the implementation side, we have made use of BRDF Explorer, ${ }^{15}$ which we have extended to incorporate the computation of moments.

\subsection{Definitions}

Moments are statistical quantities that characterize the shape of a distribution. For a scalar distribution $f$ defined over a domain of arbitrary dimension, moments are quantitative measures of the shape of $f$ defined by:

$$
\boldsymbol{\mu}_{k}[f]=\int_{X} \underbrace{\mathbf{x} \otimes \cdots \otimes \mathbf{x}}_{k \text { factors }} f(\mathbf{x}) d \mathbf{x}
$$

where $k$ is the moment order, $X$ is the domain of definition of $f$ and $\otimes$ denotes a tensor product. As a result, a moment of order $k$ is a tensor of dimension $k+1$ : a scalar at order 0 , a vector at order 1 , a matrix at order 2 , etc. In particular, since $f$ is a distribution function, its 0 th order moment is given by $\boldsymbol{\mu}_{0}[f]=1$.

Now, for a scalar distribution defined over a $2 \mathrm{D}$ domain, we write $\mathbf{x}=(x, y)$ and define:

$$
\mu_{n, m}[f]:=\mathrm{E}_{f}\left[x^{n} y^{m}\right]=\iint_{X} x^{n} y^{m} f(x, y) d x d y,
$$

which corresponds to a scalar coefficient of the moment tensor of order $k=n+m$. In the following, we mostly make use of Equation 3 instead of Equation 2 as it makes our computations more explicit.

BRDF slices are scalar functions defined on a 2D hemispherical domain, which we write $\rho\left(\boldsymbol{\omega}_{o}^{\star}, \boldsymbol{\omega}_{i}\right): \Omega_{\mathbf{n}} \rightarrow \mathbb{R}^{+}$, where the view direction is starred to emphasize that it is fixed, and $\mathbb{R}^{+}$either denotes reflectance for a color channel or the average reflectance over colors. It is important to note that such a BRDF slice is not a distribution function in the general case, since its 0th order moment will likely be not equal to 1 . We will thus first normalize a BRDF slice by its 0th order moment when computing moments of order $k \geq 1$ below.

Computing 2D moments of a BRDF slice for a given view direction raises two complications. First, BRDF slices may exhibit a combination of multiple components, at least two in common situations: diffuse and specular. However, moments are good descriptors only for unimodal functions; computed statistics are not meaningful otherwise. Consequently, we explain in Section 2.2 how to separate slices into basic components prior to analysis. Second, using a classical parametrization in terms of elevation and azimuth angles for $\Omega$ forbids the computation 


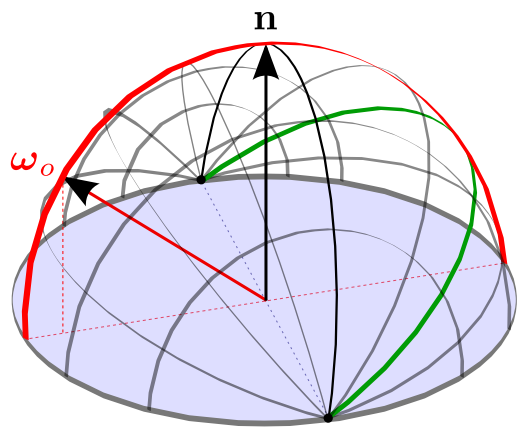

(a) Parametrization isolines

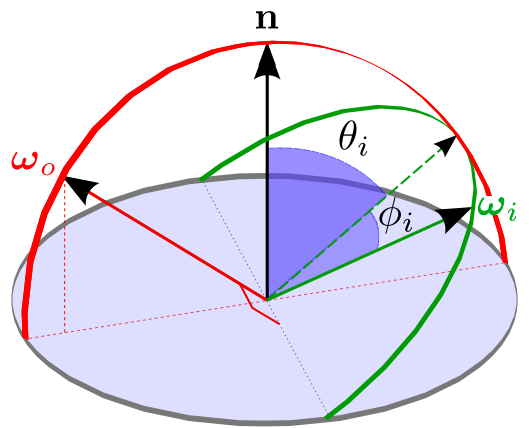

(b) Parametrization angles

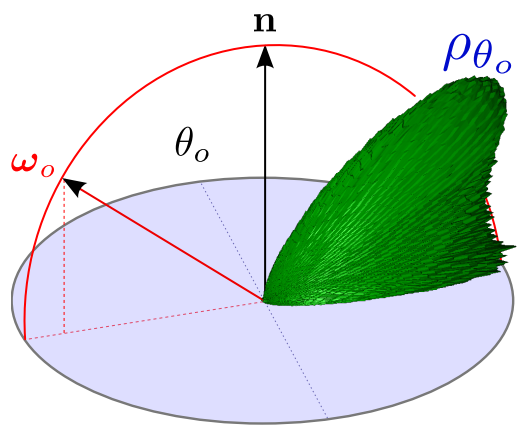

(c) 2D BRDF slice

Figure 2. (a) Our parametrization of the hemisphere has poles orthogonal to the view direction $\boldsymbol{\omega}_{o}$, which minimizes distortions in the scattering plane (in red). (b) It maps a pair of angles $\left(\theta_{i}, \phi_{i}\right) \in\left[-\frac{\pi}{2}, \frac{\pi}{2}\right]^{2}$ to a direction $\boldsymbol{\omega}_{i} \in \Omega_{\mathbf{n}}$. (c) A $2 \mathrm{D}$ BRDF slice $\rho_{\theta_{o}}$ is directly defined in our parametrization through this angular mapping.

of some moments. Indeed, it would make the domain periodic along the azimuthal dimension, say $y$ in Equation 3, which is problematic since $y^{m}$ is anti-symmetric for any odd $m$. To deal with this issue, we introduce in Section 2.3 a planar, view-centered parametrization for a BRDF slice.

\subsection{BRDF decomposition \& selection}

We relied on a simple heuristic method to separate diffuse and specular components in BRDF data. We have observed that many BRDFs from the MERL database display a near constant (i.e. close to Lambertian) diffuse component. Such a perfect diffuse component can be extracted using a simple thresholding scheme: we sample the BRDF at a viewing elevation of 45 degrees and retrieve the minimum reflectance value. We then remove this constant from the data in order to obtain its specular component, on which we ground our study.

However, even after removing a Lambertian component from BRDF data, some materials still show multimodal behaviors. We simply remove these BRDFs from our set manually, leaving a total of 40 unimodal BRDFs that we list in Appendix 5. They still span a wide range of appearances, from rough to shiny materials. A finer BRDF decomposition would allow us to study a larger subset of BRDFs from the MERL database. This is left to future work and discussed in Section 4.

\subsection{View-centered parametrization}

A solution to deal with the periodicity of the hemispherical domain would be to compute 3D moments using cartesian coordinates as done by Ghosh et al. ${ }^{8}$ However, this would not only make analysis harder (relying on 3D tensors), but it would also unnecessarily introduce distortions at grazing angles, where hemispherical and Euclidean distances differ markedly. An alternative would be to rely on statistics based on lighting elevation, as done by Havran et $\mathrm{al}^{9}$ for the purpose of material characterization. Unfortunately, this approach is not adapted to our purpose since it reduces a priori the complexity of BRDFs by using a 1D analysis. Instead, we compute moments using a planar 2D parametrization that introduces as little distortion as possible for isotropic BRDFs.

Most of the energy of a BRDF slice is concentrated around the scattering plane spanning $\boldsymbol{\omega}_{o}$ and $\mathbf{n}$. We thus convert a BRDF slice using a view-centered angular parametrization with poles orthogonal to the view direction $\omega_{o}$ (see Figure 2a). This has the advantage of minimizing distortions around the scattering plane, where most of the BRDF energy is concentrated. Formally, we specify it by a mapping $m:\left[-\frac{\pi}{2}, \frac{\pi}{2}\right]^{2} \rightarrow \Omega_{\mathbf{n}}$, given by:

$$
m(\theta, \phi)=(\sin \theta \cos \phi, \sin \phi, \cos \theta \cos \phi),
$$

where $\phi$ is the angle made with the scattering plane, and $\theta$ the angle made with the normal in the scattering plane. This is illustrated for $\boldsymbol{\omega}_{i}=m\left(\theta_{i}, \phi_{i}\right)$ in Figure $2 \mathrm{~b}$.

The projection of a BRDF slice into our parametrization is then defined by:

$$
\rho_{\theta_{o}}\left(\theta_{i}, \phi_{i}\right):=\rho\left(m\left(\theta_{o}, 0\right), m\left(\theta_{i}, \phi_{i}\right)\right),
$$



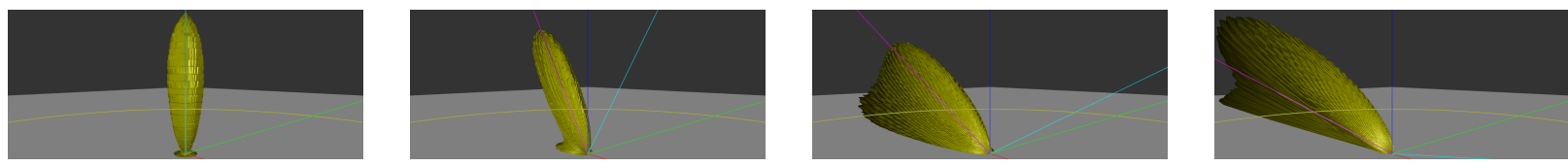

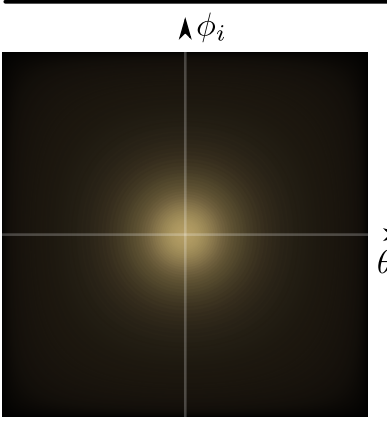

$\theta_{0}=0^{\circ}$

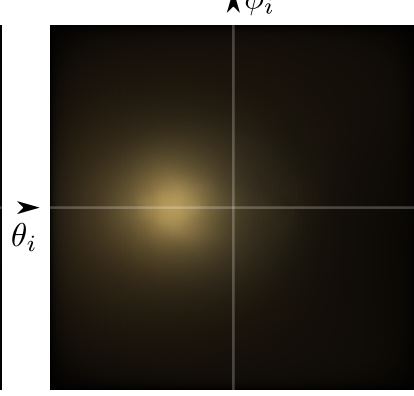

$\theta_{o}=30^{\circ}$

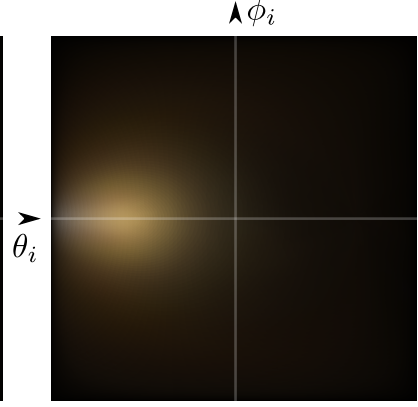

$\theta_{o}=60^{\circ}$

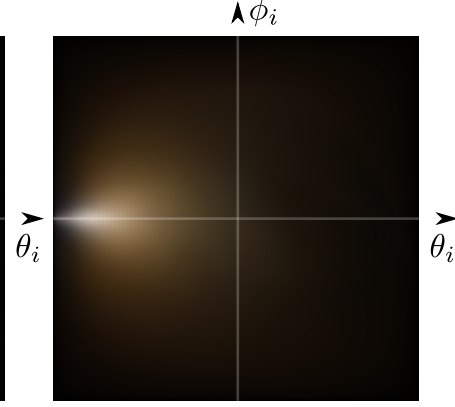

$\theta_{o}=80^{\circ}$

Figure 3. Top row: 3D visualization of four slices of the gold-paint BRDF at increasing viewing angles. Bottom row: the same BRDF slices visualized in our view-dependent parametrization.

where $\theta_{i}$ and $\phi_{i}$ are the coordinates of $\omega_{i}$ in our parametrization. Slices projected in our parametrization for a few viewing elevations are shown in Figure 3. We are now ready to compute the 2D moments of a BRDF slice $\rho_{\theta_{o}}$ in our parametrization, as functions of viewing elevation $\theta_{o}$. We call such functions moment profiles.

\subsection{Moment profiles}

For a dense set of viewing elevations, we compute 2D moments up to order 4 by discretizing Equation 3 . In practice, we sample the $\theta_{o}$ dimension uniformly in angles, each sample yielding a projected BRDF slice as shown in Figure 3. We then use a Monte Carlo estimator to evaluate the 2D moments for each of these slices:

$$
\mu_{n, m}[\rho]\left(\theta_{o}\right) \approx \frac{\pi^{2}}{N} \sum_{i=1}^{N} \theta_{i}^{n} \phi_{i}^{m} \rho_{\theta_{o}}\left(\theta_{i}, \phi_{i}\right),
$$

where $\mathbf{x}_{i}=\left(\theta_{i}, \phi_{i}\right)$ is the $i$ th randomly generated sample in the slice, and $N$ is the number of samples.

In the following, we present moment profiles computed at increasing orders, as shown in Figures 4 and 5 . For the sake of clarity, we will omit the dependence on $\theta_{o}$ both for BRDF slices and 2D moments.

Energy. If $\rho$ were a distribution function, we would have a constant $\mu_{0}[\rho]=1$ for any view elevation $\theta_{o}$. However, in general, this will not be the case as most real materials absorb at least some of the incident lighting. The 0th order moment profile thus represents an important property of a BRDF: the reflected energy as a function of $\theta_{o}$, which we denote $\alpha$. It is similar to the directional albedo, but differs in two ways: it does not take into account the cosine term, and is defined in our parametrization. As may be seen in Figure 4(a) where we show $\alpha$ for all of our selected BRDFs (red channel only), it looks mostly constant except near grazing angles.

Mean. For moments of order 1 and higher, we must normalize by the 0th order moment as explained in section 2.1. We thus write $\bar{\rho}=\frac{\rho}{\alpha}$. The coefficients of the 1st order moment vector are now given by $\mu_{n, m}[\bar{\rho}]=$ $E_{\bar{\rho}}\left[\theta_{i}^{n} \phi_{i}^{m}\right]$ for $n+m=1$. The resulting 1st order moment profiles characterize the mean direction of a BRDF slice via a $2 \mathrm{D}$ vector in our parametrization. The profile for $\mu_{1,0}$ (the coordinate along $\theta_{i}$ ) is shown in Figure 4(b): our selected BRDFs exhibit profiles that have different slopes, with deviations from a line occurring toward grazing angles. In contrast the profile of $\mu_{0,1}$ (the coordinate along $\phi_{i}$ ), as shown in Figure $5($ a), remains close to zero for all values of $\theta_{o}$. We explain the origin of such near-zero moment profiles in Section 2.5. 


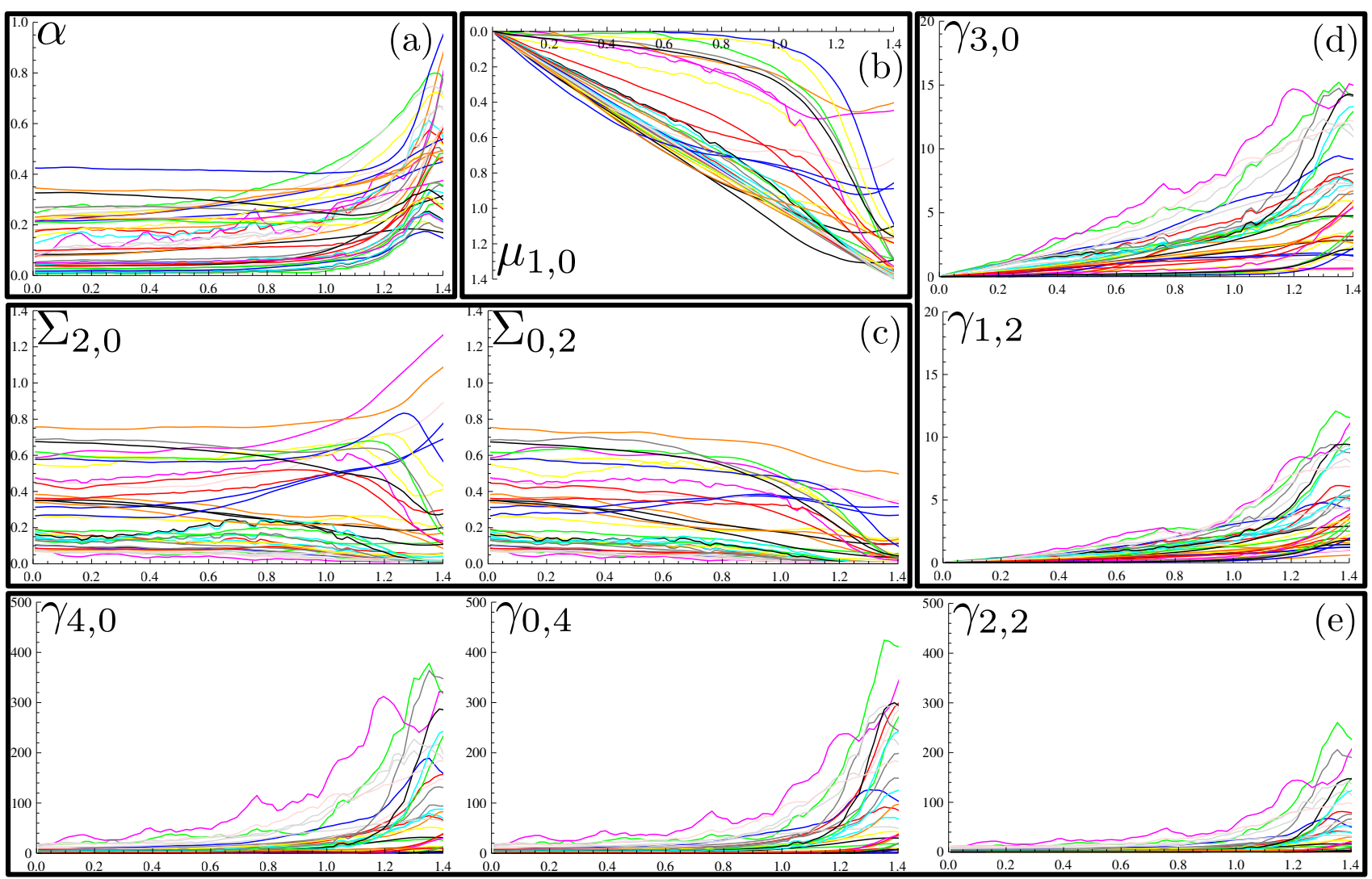

Figure 4. Moment profiles computed from our selected BRDFs are shown at increasing moment orders from (a) to (e).

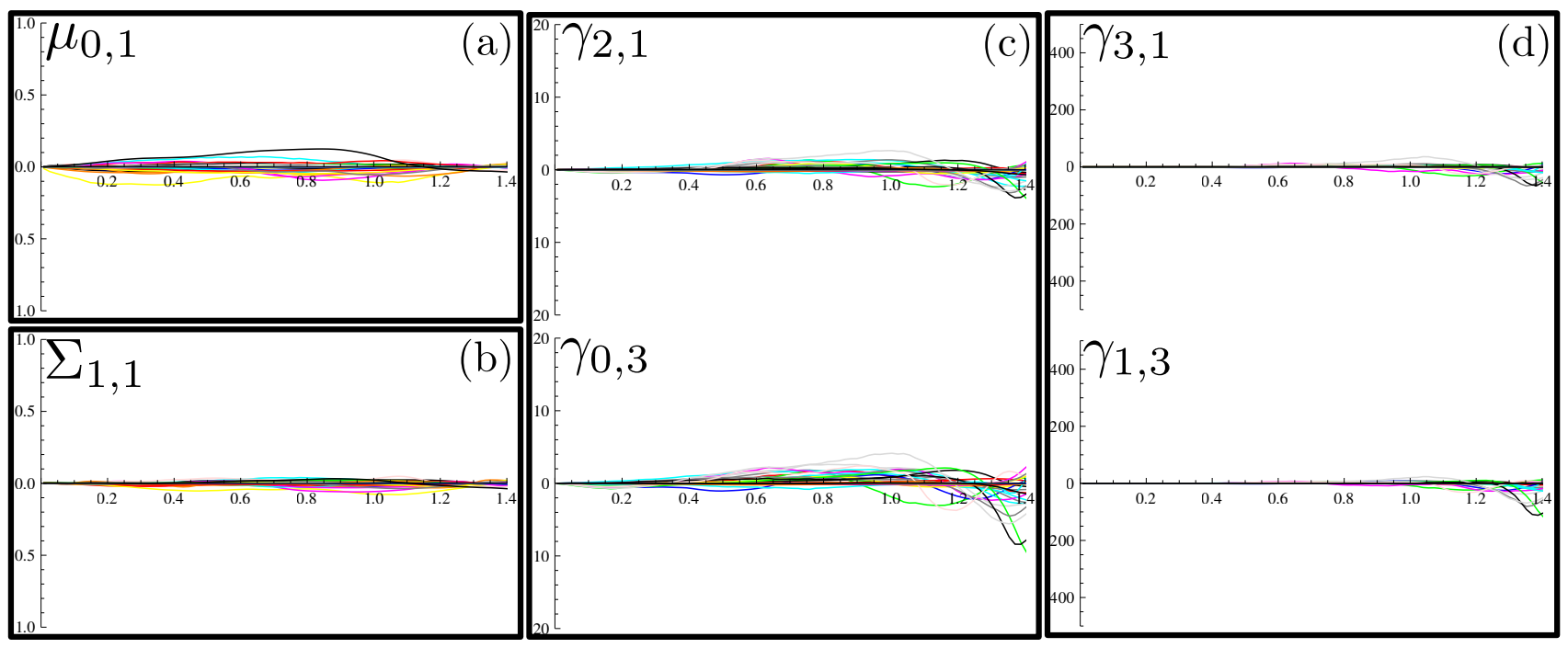

Figure 5. Near-zero moment profiles occur at all orders and are likely due to BRDF slice symmetry.

Co-variance. It is defined as the centralized moment matrix $\Sigma$ of order 2 , which consists of moments of $\bar{\rho}$ centered on its mean. In our case, since $\mu_{0,1} \approx 0$, the coefficients of the co-variance matrix may be written using a slightly simpler formula: $\Sigma_{n, m}[\bar{\rho}]=E_{\bar{\rho}}\left[\left(\theta_{i}-\mu_{\theta}\right)^{n} \phi_{i}^{m}\right]$ for $n+m=2$, where $\mu_{\theta}:=\mu_{1,0}$. This matrix characterizes how the BRDF slice is spread around its mean, with larger values in either dimension implying larger spread. The profiles for the diagonal coefficients $\Sigma_{2,0}$ and $\Sigma_{0,2}$ are shown in Figure 4 (c): our selected BRDFs exhibit profiles of different variances, with slight deviations from the average occurring toward grazing 
viewing angles. The off-diagonal coefficient $\Sigma_{1,1}$ remains close to zero as shown in Figure 5(b).

Co-skewness and co-kurtosis. They are defined as the standardized moment tensors of order 3 and 4 respectively. Standardized moments are computed by both centering $\bar{\rho}$ on its mean and scaling it by respective variances. Since in our case $\Sigma_{1,1} \approx 0$, we may write standardized moments using $\gamma_{n, m}[\bar{\rho}]=E_{\bar{\rho}}\left[\left(\frac{\theta_{i}-\mu_{\theta}}{\sigma_{\theta}}\right)^{n}\left(\frac{\phi_{i}}{\sigma_{\phi}}\right)^{m}\right]$, where $\sigma_{\theta}=\sqrt{\Sigma_{2,0}}$ and $\sigma_{\phi}=\sqrt{\Sigma_{0,2}}$. The coefficients of the co-skewness and co-kurtosis tensors are then given by $\gamma_{n, m}[\bar{\rho}]$ for $n+m=3$ and $n+m=4$ respectively. It is common to use the modern definition of kurtosis, also called excess kurtosis, which is is equal to 0 for a Normal distribution. In our case (i.e., with $\mu_{0,1}=0$ and $\Sigma_{1,1}=0$ ), it can be shown that excess kurtosis coefficients are given by $\gamma_{4,0}-3, \gamma_{3,1}, \gamma_{2,2}-1, \gamma_{1,3}$ and $\gamma_{0,4}-3$. For simplicity, we will make an abuse of notation and refer to excess kurtosis coefficients as $\gamma_{n, m}[\bar{\rho}]$ for $n+m=4$.

The co-skewness tensor characterizes asymmetries of the BRDF slices in different dimensions. The profile for two of its coefficients, $\gamma_{3,0}$ and $\gamma_{1,2}$, are shown in Figures 4 (d): our selected BRDFs exhibit increasing skewness toward grazing angles, which are more pronounced for $\gamma_{3,0}$ than $\gamma_{1,2}$. The other two profiles are close to zero and shown in Figures 5(c). The co-kurtosis tensor characterizes the peakedness of the BRDF slice in different dimensions. The profile for three of its coefficients, $\gamma_{4,0}, \gamma_{2,2}$ and $\gamma_{0,4}$, are shown in Figures 4(e): our selected BRDFs exhibit roughly constant profiles, with deviations occurring at grazing angles, which are most pronounced in $\gamma_{4,0}$. The other two profiles are close to zero and shown in Figures $5(\mathrm{~d})$.

\subsection{Discussion}

We have mentioned throughout Section 2.4 that a number of moment profiles are near-zero for all of our selected BRDFs. By inspecting these moment profiles in Figure 5, it appears that all moments where $m$ is odd seem to be close to 0 . Since moments with odd $m$ correspond to anti-symmetric functions in the $\phi_{i}$ dimension, this strongly suggests that near-zero profiles are due to the near-symmetry of most isotropic BRDFs about the scattering plane (i.e. along $\phi_{i}$ ), as seen in Figure 3.

The value at $\theta_{o}=0$ (incident view) is also of interest for all moment orders. At orders 1 and 3 , moments for all materials start with a zero value. At orders 2 and 4, moments for each material along $\theta_{i}$ or $\phi_{i}$ axis start at the same value. We believe the reason for this is that slices of isotropic BRDFs are near radially symmetric around the normal (the origin in our parametrization) at incidence.

Lastly, all materials tend to exhibit deviations with respect to a simple profile toward grazing viewing angles. This might be due to specific modes of reflectance such as asperity scattering ${ }^{16}$ coming in to reshape the BRDF slice. However, the effect seems stronger for moments along $\theta_{i}$ than for those along $\phi_{i}$. We thus conjecture that such grazing-angle deviations are due in part to the clamping of directions by hemispherical boundaries. Indeed, such a clamping will have more influence at grazing angles in directions parallel to $\theta_{i}$ (see Figure 3 ).

\section{STATISTICAL ANALYSIS}

The previous section has provided us with a characterization of BRDF data in terms of statistical moment profiles up to order 4 . We now wish to understand how such BRDF properties influence image properties when used in renderings, this time only considering moments up to order 2 . The main reason for this choice is that higher-order moments are all computed based on standardized moments, which are based on moments of order 1 and 2. Their understanding hence directly depends on the understanding of lower-order moments.

We first introduce a BRDF slice model based on moment profiles and incorporate it in the reflected radiance equation. We then conduct a local Fourier analysis that yields direct relationships between reflected radiance and BRDF/lighting moments around a fixed view elevation. Lastly, we fit analytic functions to moment profiles and study their correlation to understand how BRDF properties appear in the image for a varying view elevation. 


\subsection{BRDF slice model.}

Before we can study how BRDF properties might be translated in terms of image properties, we need to define a moment-based BRDF slice model for use in Equation 1. We would like to point out that this model does not ensure reciprocity, and shall thus not be used outside of this statistical analysis. Our model is defined by:

$$
\rho_{\theta_{o}}\left(\theta_{i}, \phi_{i}\right)=\alpha\left(\theta_{o}\right) g_{\sigma_{\theta}\left(\theta_{o}\right)}\left(\theta_{i}-\mu_{\theta}\left(\theta_{o}\right)\right) g_{\sigma_{\phi}\left(\theta_{o}\right)}\left(\phi_{i}\right),
$$

where $g_{\sigma}$ is a $1 \mathrm{D}$ Gaussian of variance $\sigma^{2}$. Both gaussians correspond to normal distributions that have been rescaled to guarantee energy conservation on our parametrization domain. The scaling term is given by:

$$
A=\int_{-\frac{\pi}{2}-t_{0}}^{\frac{\pi}{2}-t_{0}} e^{-\frac{t^{2}}{2 \sigma^{2}}} \mathrm{~d} t=\sqrt{\frac{\pi \sigma^{2}}{2}}\left[\operatorname{erf}\left(\frac{\pi / 2-t_{0}}{\sqrt{2 \sigma^{2}}}\right)-\operatorname{erf}\left(-\frac{\pi / 2-t_{0}}{\sqrt{2 \sigma^{2}}}\right)\right],
$$

where we have restricted the domain to $\left[-\frac{\pi}{2}, \frac{\pi}{2}\right]$ and centered it on $t_{0}$. This accommodates both Gaussians with $t_{0}=\mu_{\theta}\left(\theta_{o}\right)$ in one case, and $t_{0}=0$ in the other.

Our model is separable, which is a consequence of the symmetry of BRDF slices around the scattering plane. Moreover, we assume that $\sigma_{\theta}(0)=\sigma_{\phi}(0)$ to ensure the BRDF slice is radially symmetric at viewing incidence.

\subsection{Local Fourier analysis.}

Our analysis begins with a change of variable in Equation 1 using our parametrization. Analysis is performed in a local tangent frame for simplicity, with the domain of integration being the input space of $m$ :

$$
R\left(\theta_{o}, \phi_{o}\right)=\int_{-\frac{\pi}{2}}^{\frac{\pi}{2}} \int_{-\frac{\pi}{2}}^{\frac{\pi}{2}} \rho_{\theta_{o}}\left(\theta_{i}, \phi_{i}\right) L\left(m\left(\theta_{i}, \phi_{i}\right)\right) \cos \theta_{i} \cos ^{2} \phi_{i} d \theta_{i} d \phi_{i}
$$

where the 3rd coordinate of $\omega_{i}=m\left(\theta_{i}, \phi_{i}\right)$ (given by $\cos \theta_{i} \cos \phi_{i}$ according to Equation 4) stands for the cosine term in tangent space. Replacing $\rho_{\theta_{o}}$ with our moment-based BRDF slice model (Equation 7) yields:

$$
R\left(\theta_{o}, \phi_{o}\right)=\alpha\left(\theta_{o}\right) \int_{-\frac{\pi}{2}}^{\frac{\pi}{2}} g_{\sigma_{\theta}\left(\theta_{o}\right)}\left(\theta_{i}-\mu_{\theta}\left(\theta_{o}\right)\right)\left(\int_{-\frac{\pi}{2}}^{\frac{\pi}{2}} g_{\sigma_{\phi}\left(\theta_{o}\right)}\left(\phi_{i}\right) L\left(m\left(\theta_{i}, \phi_{i}\right)\right) \cos \theta_{i} \cos ^{2} \phi_{i} d \phi_{i}\right) d \theta_{i} .
$$

Now, since our BRDF slice model is separable in $\theta_{i}$ and $\phi_{i}$, we may pursue our study in either dimension independently. Let us focus on $\theta_{i}$. If we fold in the integral of terms over $\phi_{i}$ and cosines and write:

$$
L_{\phi}\left(\theta_{i}\right)=\int_{-\frac{\pi}{2}}^{\frac{\pi}{2}} g_{\sigma_{\phi}\left(\theta_{o}\right)}\left(\phi_{i}\right) L\left(m\left(\theta_{i}, \phi_{i}\right)\right) \cos \theta_{i} \cos ^{2} \phi_{i} d \phi_{i}
$$

then Equation 10 turns into a 1D integral of the form:

$$
R\left(\theta_{o}, \phi_{o}\right)=\alpha\left(\theta_{o}\right) \int_{-\frac{\pi}{2}}^{\frac{\pi}{2}} g_{\sigma_{\theta}\left(\theta_{o}\right)}\left(\theta_{i}-\mu_{\theta}\left(\theta_{o}\right)\right) L_{\phi}\left(\theta_{i}\right) d \theta_{i}
$$

Our next step is to approximate this 1D integral with a convolution. To this end, we make local approximations of moment profiles in a $1 \mathrm{D}$ angular window around $\theta_{o}$. We assume the energy and variance to be locally constant: $\alpha\left(\theta_{o}+t\right) \approx \alpha\left(\theta_{o}\right)$ and $\sigma_{\theta}^{2}\left(\theta_{o}+t\right) \approx \sigma_{\theta}^{2}\left(\theta_{o}\right)$. For the mean profile, we rather make use of a first-order approximation: $\mu_{\theta}\left(\theta_{o}+t\right) \approx \mu_{\theta}\left(\theta_{o}\right)+\left.\frac{d \mu_{\theta}}{d t}\right|_{\theta_{o}} t$. As a result, $R$ may be approximated by a $1 \mathrm{D}$ convolution of the form:

$$
R\left(\theta_{o}+t, \phi_{o}\right) \approx \alpha\left(g_{\sigma_{\theta}} \otimes L_{\phi}\right)\left(\theta_{o}+t\right), \text { with } t \in[-\epsilon,+\epsilon],
$$

where we have dropped the dependencies of both $\alpha$ and $\sigma_{\theta}$ on $\theta_{o}$ since they are assumed locally constant.

In Fourier space, this convolution turns into the following product:

$$
\mathcal{F}[R](\xi) \approx \alpha \mathcal{F}\left[g_{\sigma_{\theta}}\right](\xi) \mathcal{F}\left[L_{\phi}\right](\xi),
$$

where $\xi$ is the Fourier variable corresponding to $t$. Note that Fourier shifts $e^{i \theta_{o}}$ due to the centering on $\theta_{o}$ cancel out since they appear on both sides of the equation. Equation 12 bears similarities with previous work, ${ }^{11,13}$ with the difference that our approach provides direct connections with moments thanks to our BRDF slice model. 


\subsection{Relationships between moments.}

An important property of moments is that they are directly related to the Fourier transform of a function $f$ by: ${ }^{7}$

$$
\mathcal{F}[f](\xi)=\sum_{k} \frac{(i \xi)^{k}}{k !} \mu_{k}[f]
$$

where $\mu_{k}[f]$ is the $k$-th moment of $f$. We thus re-write Equation 13 as a product of moment expansions:

$$
\mathcal{F}[R](\xi)=\alpha\left(\sum_{k} \frac{(i \xi)^{k}}{k !} \mu_{k}\left[g_{\sigma_{\theta}}\right]\right)\left(\sum_{l} \frac{(i \xi)^{l}}{l !} \mu_{l}\left[L_{\phi}\right]\right) .
$$

To establish relationships between moments, we extract the moments from $\mathcal{F}[R]$ using its own moment expansion. This is done by differentiating $\mathcal{F}[R]$ at $\xi=0:{ }^{7}$

$$
\begin{aligned}
& \mu_{0}[R]=\mathcal{F}[R](0) \\
& \mu_{1}[R]=\operatorname{Im}\left\{\frac{\mathrm{d} \mathcal{F}[R]}{\mathrm{d} \xi}(0)\right\} \\
& \mu_{2}[R]=-\operatorname{Re}\left\{\frac{\mathrm{d}^{2} \mathcal{F}[R]}{\mathrm{d} \xi^{2}}(0)\right\} .
\end{aligned}
$$

Next, we expand Equation 15 and its derivatives at $\xi=0$ and plug them inside Equations 16 through 18:

$$
\begin{aligned}
\mu_{0}[R] & =\alpha \mu_{0}\left[g_{\sigma_{\theta}}\right] \mu_{0}\left[L_{\phi}\right], \\
\mu_{1}[R] & =\mu_{1}\left[g_{\sigma_{\theta}}\right]+\mu_{1}\left[L_{\phi}\right], \\
\mu_{2}[R] & =\mu_{2}\left[g_{\sigma_{\theta}}\right]+\mu_{2}\left[L_{\phi}\right]+2 \mu_{1}\left[g_{\sigma_{\theta}}\right] \mu_{1}\left[L_{\phi}\right] .
\end{aligned}
$$

Since $g_{\sigma_{\theta}}$ is normalized, $\mu_{0}\left[g_{\sigma_{\theta}}\right]=1$. However, $\mu_{0}[R] \neq 1$ in the general case, and we must normalize moments of order 1 and 2 before going further. As in Section 2.4, we write $\bar{R}=R / \mu_{0}[R]$, which yields $\mu_{k}[\bar{R}]=\frac{\mu_{k}[R]}{\mu_{0}[R]}$. It can then be easily shown that Equations 20 and 21 remain valid after normalization.

Lastly, we write the variance of $\bar{R}$ in terms of moments: $\operatorname{Var}[\bar{R}]=\mu_{2}[\bar{R}]-\mu_{1}^{2}[\bar{R}]$. After carrying out simplifications, we get: $\operatorname{Var}[\bar{R}]=\operatorname{Var}\left[\bar{g}_{\sigma_{\theta}}\right]+\operatorname{Var}\left[\bar{L}_{\phi}\right]$. Putting it all together, we obtain the following moment relationships for a given viewing elevation $\theta_{o}$ :

$$
\begin{aligned}
\mu_{0}[R]\left(\theta_{o}\right) & =\alpha\left(\theta_{o}\right) \mu_{0}\left[L_{\phi}\right]\left(\theta_{o}\right), \\
\mathrm{E}[\bar{R}]\left(\theta_{o}\right) & =\mu_{\theta}\left(\theta_{o}\right)+\mathrm{E}\left[\bar{L}_{\phi}\right]\left(\theta_{o}\right), \\
\operatorname{Var}[\bar{R}]\left(\theta_{o}\right) & =\sigma_{\theta}^{2}\left(\theta_{o}\right)+\operatorname{Var}\left[\bar{L}_{\phi}\right]\left(\theta_{o}\right),
\end{aligned}
$$

where we have used $\mathrm{E}\left[\bar{g}_{\sigma_{\theta}}\right]\left(\theta_{o}\right)=\mu_{\theta}\left(\theta_{o}\right)$ and $\operatorname{Var}\left[\bar{g}_{\sigma_{\theta}}\right]\left(\theta_{o}\right)=\sigma_{\theta}^{2}\left(\theta_{o}\right)$. The reasoning is similar when studying the integral along $\phi_{i}$, in which case we must define a $L_{\theta}$ term analogous to $L_{\phi}$. We then obtain similar moment relationships, except in this case $\mathrm{E}\left[\bar{g}_{\sigma_{\phi}}\right]=0, \operatorname{Var}\left[\bar{g}_{\sigma_{\phi}}\right]=\sigma_{\phi}^{2}$, and $L_{\phi}$ is replaced by $L_{\theta}$.

\subsection{Fitting \& correlation}

In the previous section, we have derived moment relationships as the result of local approximations of moment profiles around a fixed viewing elevation. In this section, we investigate global approximations of moment profiles through the fitting of analytic functions for the entire viewing range. Our goal here is to better understand similarities across materials for a same moment order, as well as correlations among different orders.

Naturally, we will less focus on fitting accuracy than on concision: a few profile parameters are necessary if we wish to compare profiles across many measured materials. Regarding color channels, we have tried fitting them separately, or fitting their average for each slice directly. We only report fits based on averages since for our selected materials we found differences across fits for different color channels to be negligible. It must be noted that for the energy, profiles for each color channel will obviously be different; however they are merely 

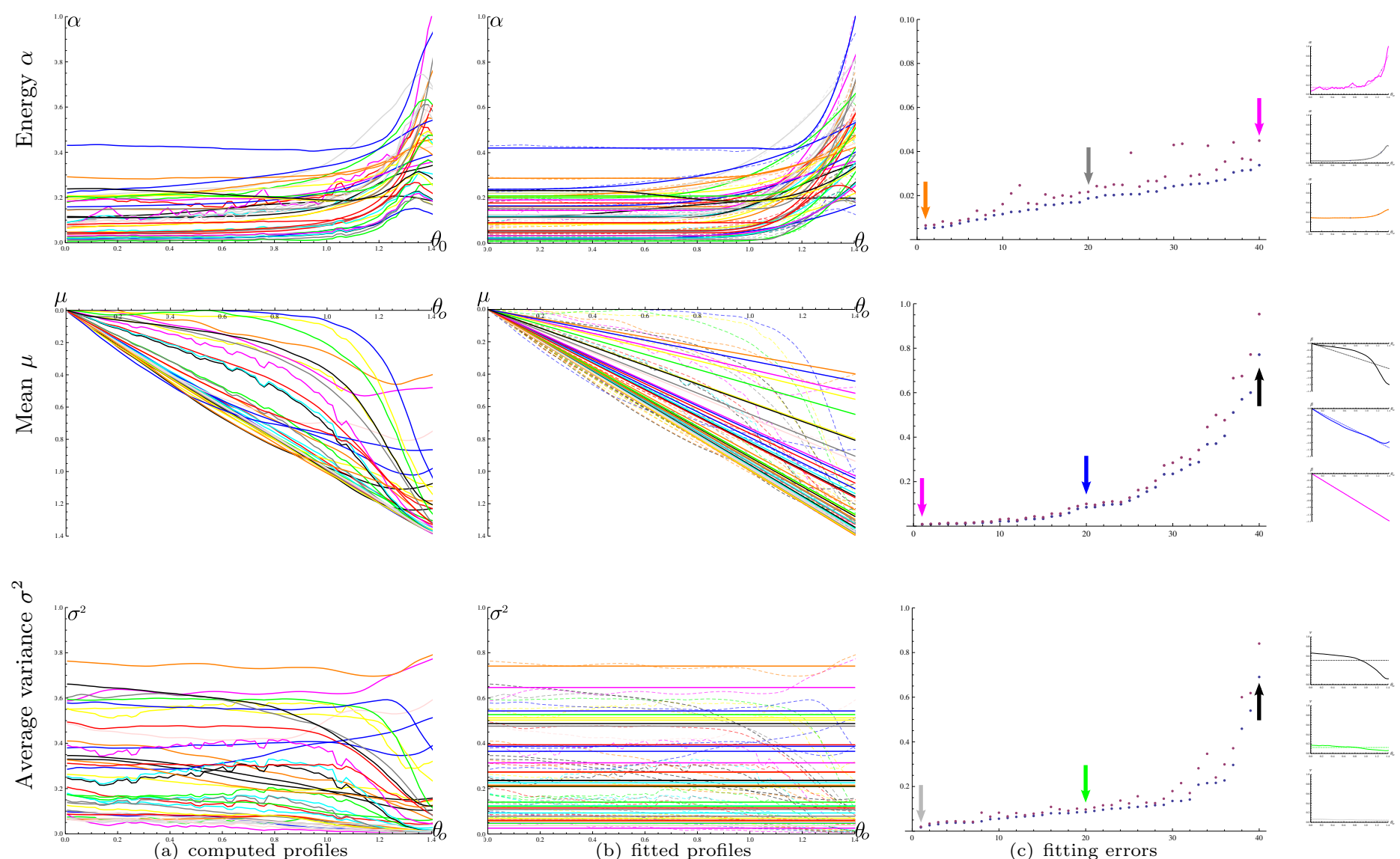

Figure 6. We fitted the moment profiles (from top to bottom: energy, mean and average variance) from the selected materials list. We provide in each column the computed moment profiles (a), the fitted profiles (b), and the corresponding fitting errors (c). The error of our fits is computed using both the Mean Absolute Error (MAE in blue) and the Root Mean Square Error (RMSE in purple). The small inset profiles correspond to worse, intermediate and best fits.

offseted with respect to each other. It is thus also reasonable to fit their average since we are mostly interested in the shape of profile functions.

Figure 6 shows the fitting results for the energy, mean and variance profiles, as detailed below. It shows computed profiles, fitted profiles, fitting errors and representative "best", "intermediate" and "worse" fits. We introduce our choices of analytical function for each moment order in turn.

Energy. As seen in Figure 6, the energy profiles $\alpha\left(\theta_{o}\right)$ for our selection of BRDFs exhibit a constant behavior up to grazing angles, at which point they tend to increase progressively. We model this profile with $\alpha\left(\theta_{o}\right) \approx$ $\tilde{\alpha}\left(\theta_{o}\right)=\tilde{\alpha}_{b}+\tilde{\alpha_{s}}\left(\theta_{o}\right)$ where $\tilde{\alpha_{b}}$ represents the constant base energy and $\tilde{\alpha}_{s}$ is an Hermite spline that deals with the increase in energy. The Hermite spline itself is modeled with two knots defined by their angular position $\theta_{0}$, $\theta_{1}$, energy value $\alpha\left(\theta_{0}\right), \alpha\left(\theta_{1}\right)$ and slope $m_{0}, m_{1}$ (see Figure $7(\mathrm{a})$ ).

We use a non-linear optimization to fit these parameters to each energy profile, using $\theta_{0}=45$ and $\theta_{1}=75$ degrees as initial values, and constraining $m_{0}=0$ to reproduce the constant profile far from grazing angles. We first fit knot positions independently for each material, which yields an average $\theta_{0}$ of 35.3 degrees with a standard deviation of 14.8 , and an average $\theta_{1}$ of 71.5 degrees with a standard deviation of 5.2. Combined with the observation that $\alpha_{1}>\alpha_{0}$ in all our materials, this suggests that all our materials exhibit an energy boost confined to grazing viewing angles. We then fit the same knot positions for all our materials; this yields $\theta_{0}=38.7$ degrees and $\theta_{1}=69.9$ degrees, which confirms the grazing energy boost tendency. 


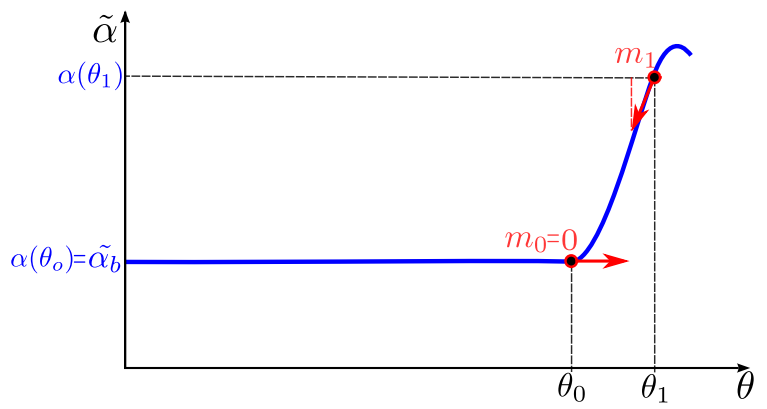

(a) Energy profile function $\tilde{\alpha}$

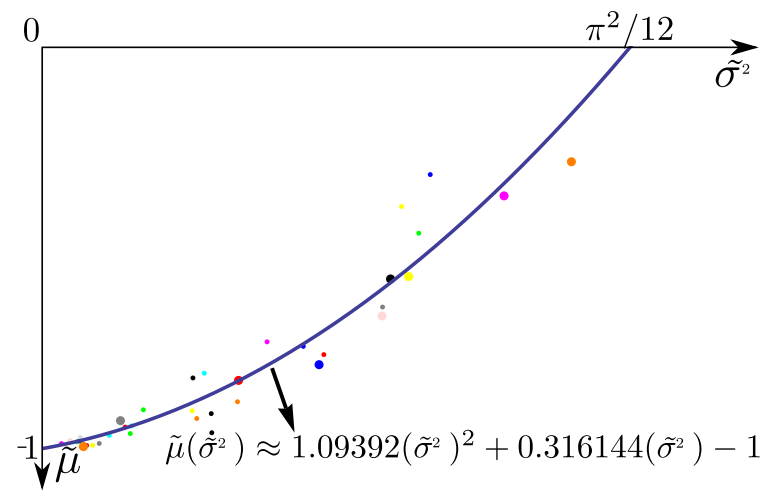

(b) Correlation between $\tilde{\mu}$ and $\tilde{\sigma}^{2}$

Figure 7. (a) The energy profile $\tilde{\alpha}$ may exhibit a silhouette effect, which we model by a Hermite spline starting at $\theta_{0}$ with $m_{0}=0$, and ending at $\theta_{1}$ with a fitted $m_{1}$. (b) We fit the correlation between the mean slope $\tilde{\mu}$ and the average variance $\tilde{\sigma}^{2}$ using a quadratic function.

Mean. Concerning the mean profile $\mu_{\theta}\left(\theta_{o}\right)$, the vast majority of cases show a linear tendency, with slopes proportional to the specularity of the material. Moreover, all profile functions go through the origin, as previously observed in Section 2.5. This suggests that a linear fit $\mu_{\theta}\left(\theta_{o}\right) \approx \tilde{\mu} \theta_{o}$ is appropriate for representing this behavior. We fit the single slope parameter $\tilde{\mu}$ using a least-squares optimization, which always leads to a negative value due to our choice of parametrization (e.g., the mirror direction is given by $\tilde{\mu}=-1$ ). It is interesting to observe that our materials exhibit mean slopes nearly spanning the entire range from -1 to 0 .

Variance. We have observed in Section 2.5 that $\sigma_{\theta}^{2}(0) \approx \sigma_{\phi}^{2}(0)$, which is likely to be due to radial symmetry at viewing incidence. Our data also reveals that the deviations from a constant behavior observed around grazing angles tend to increase for $\sigma_{\theta}^{2}$ and decrease for $\sigma_{\phi}^{2}$. We thus choose to study the average variance using a constant profile, hence writing $\frac{\left(\sigma_{\theta}^{2}\left(\theta_{o}\right)+\sigma_{\phi}^{2}\left(\theta_{o}\right)\right)}{2} \approx \tilde{\sigma}^{2}$. The constant parameter is obtained using a least-squares fitting as before, with values ranging from 0 for a mirror to $\frac{\pi^{2}}{12}$ for a Lambertian (see Appendix 6). Once again, our materials exhibit a large range of average variances.

Correlation. Looking at Figure 6(b), one may observe a seeming correlation between the fitted mean slope $\tilde{\mu}$ and average variance $\tilde{\sigma}^{2}$ : the lower the variance, the steeper the mean. To investigate this potential correlation, we plot one parameter against the other in Figure 7, which indeed exhibits a correlation. We thus perform a least-squares fitting with a quadratic function, using parameters of a mirror material $\left(\tilde{\mu}=-1\right.$ and $\left.\tilde{\sigma}^{2}=0\right)$ and of a Lambertian one $\left(\tilde{\mu}=0\right.$ and $\left.\tilde{\sigma}^{2}=\pi^{2} / 12\right)$ as end point constraints. We conjecture that this correlation is again due to hemispherical clamping. Because of clamping, the distribution mean is slightly offset toward incidence compared to the distribution peak, and this effect is all the more pronounced for distributions of high variance: wider distributions are clamped early on relative to narrow ones.

\subsection{Discussion}

Taken together, the results of Sections 3.3 and 3.4 provide insights on how BRDF moments influence the moments of reflected radiance, and ultimately become observable in pictures.

As an example, let's consider a distant environment illumination reflected off a sphere: in this case, each $\left(\theta_{o}, \phi_{o}\right)$ pair corresponds to a point on the sphere surface. We begin with moment relationships along the $\theta_{i}$ dimension (i.e., radial lines on the sphere). Equation 22 then tells us that the material color has a multiplicative effect $\left(\tilde{\alpha}_{b}\right)$ everywhere on the sphere, with an additional boost $\left(\tilde{\alpha}_{s}\right)$ toward the silhouette. Equations 23 and 24 show that material roughness appears through a warping $\left(\tilde{\mu} \theta_{o}\right)$ that constantly increases toward the silhouette, correlated with an overall blurring $\left(\tilde{\sigma}^{2}\right)$. In contrast, along the $\phi_{i}$ dimension (i.e., along concentric circles on a sphere), only blurring occurs, which is consistent with the symmetry of BRDF slices around the scattering plane. 
In the extreme case of a Lambertian diffuse component, there is no warping since $\tilde{\mu}=0$; for a mirror specular component, there is no blurring since $\tilde{\sigma}^{2}=0$.

Figure 8 provides an illustration of the effect of a BRDF on the image of a rendered sphere. We start from an ideal mirror BRDF in Figure 8 (a), which exhibits extreme sharpness and warping of the environment toward the silhouette as expected. We then study the effect of two BRDFs: specular-black-phenolic and pearl-paint, with renderings shown in Figures 8 (b) and (d). In both case, we focus on three points increasingly closer to the silhouette (i.e., at increasingly grazing viewing elevation). Our analysis reveals that a BRDF directly acts as an image filter whose properties are governed by statistical moments. In Figure 8 (c) and (e), we show the means and co-variance ellipses (in blue) for both BRDFs at the three picked locations. The filters corresponding to the specular-black-phenolic BRDF remain close to the evaluation position (in red), and their spread is narrow, resulting in a small blur. In contrast, the filters corresponding to the pearl-paint BRDF exhibit a stronger blur, and are offseted toward the center of the sphere for increasing viewing angles. As a result, the warping due to the BRDF is less pronounced in this case, a subtle effect of this BRDF which illustrates the impact of correlation between mean and variance.

When considering shapes more complex than spheres, we may still make similar observations by considering a surface patch on the object. However, surface curvatures will impose restrictions on the window sizes we use for establishing moment relationships on this patch. In particular, high curvatures will lead to rapid changes of the view direction in surface tangent space. In such situations, our local moment profile approximations will be valid only in small 1D windows. This suggests that the effect of BRDF moments will tend to be less noticeable on bumpy surfaces, which is consistent with existing perceptual experiments. ${ }^{17}$

Up until now, we have considered moment relationships between BRDF/lighting and reflected radiance on an object surface. To get complete relationships with image moments, we should consider the effect of viewing projection on reflected radiance moments. This will of course depend on the type of virtual sensor used, and we prefer to defer this analysis to future work. We may still anticipate that foreshortening will tend to compress radiance patterns at grazing angles. This suggests that some grazing angle effects found in moment profiles will get "squeezed" in a thin image region around the silhouette. Figure 8 illustrates this: even though filters tend to get more elongated toward silhouettes, foreshortening somewhat compensates the effect.

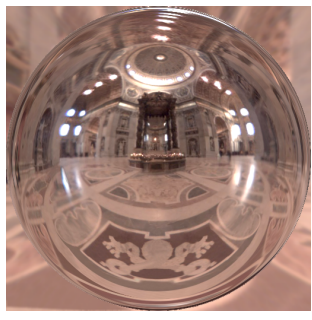

(a)

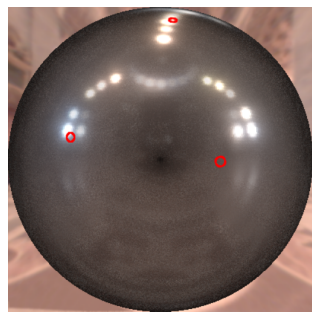

(b)

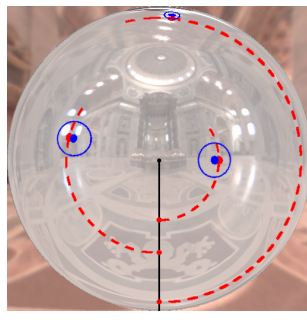

(c)

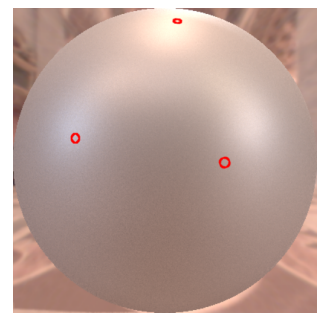

(d)

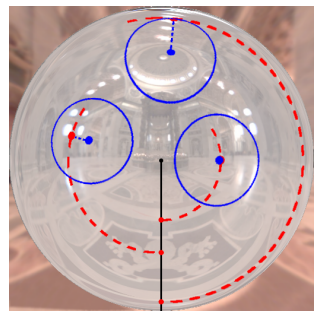

(e)

Figure 8. (a) A sphere made of an ideal mirror material rendered using the StPeter environment map. The reflected environment is extremely sharp and warped toward silhouettes. (b) A rendering using the Specular-black-phenolic BRDF. The reflected environment is slightly blurred and highly warped. This is explained in (c) by the filtering characteristics (in blue) of the BRDF at 3 different locations: the filter is narrow and remains close to the evaluation point (in red). Note that at similar viewing elevations (dashed red arcs), the filters are rotated copies of each other. (d) A rendering using the Pearl-paint BRDF. The reflected environment is this time much more blurred and exhibits less warping. This is explained in (d) by the filtering characteristics of the BRDF: the filter is wide and offset toward the center of the sphere for locations closer to the silhouette. This confirms the mean/variance correlation that we have observed in our study.

\section{GENERAL DISCUSSION}

Our statistical analysis has shown that moments of isotropic BRDF slices as functions of viewing elevation are promising properties for the study of material appearance. As opposed to previous work that relied on 3D or 1D analysis with moment orders restricted to 2 , we have demonstrated how to compute 2D moments up to order 4. Skewness and kurtosis seem to be important properties of material appearance, related to asymmetry and 
peakedness of a BRDF slice respectively. However, we have left them aside and concentrated on moments up to order 2 when deriving direct relationships with moments of lighting and reflected radiance. Together with global moment profile and correlation fitting, these helped us draw conclusions on how BRDF moments up to order 2 are conveyed in images. In a nutshell, moments of orders 0,1 and 2 induce coloring, warping and blurring of reflected radiance respectively, the last two being correlated probably due to hemispherical clamping.

We believe our approach could prove useful in a number of applications in Computer Graphics and Vision. It could be used as a way to edit measured BRDFs, by finding operators in BRDF space that preserve some moments while modifying others. Ideally, users could be granted control over a measured BRDF directly through its moment profiles. Another potential application is the estimation of material properties from photographs. Here the goal would be to recover material moments given reflected radiance moments. This will require some knowledge over lighting moments, either explicit or hypothesized. Image processing could also benefit from a moment-based approach to the editing of materials. Here the idea would be to re-filter images in ways that mimic changes in material appearance, without having to know about lighting moments in this case.

Our work requires some improvements to reach the full potential of BRDF moments. Firstly, because of our simple heuristic BRDF decomposition, we were forced to consider a subset of the MERL database. It would thus be interesting to devise clever decomposition schemes so that each component may be studied using a separate moment analysis. Second, we would like to extend our local Fourier analysis to include co-skewness and co-kurtosis tensors. As with moments up to order 2, it will be possible to study $\theta_{i}$ and $\phi_{i}$ dimensions separately, hence making the problem tractable. This will allow us to understand further the effect of a BRDF in the image, by finely characterizing the shape of image filters such as those shown in Figure 8 . Third, with the addition of moments of order 3 and 4, we would like to study potential correlations between moments of different orders. We have already observed interesting deviations from simple behaviors at grazing angles in skewness and kurtosis profiles. They may be related to known properties such as off-specular peaks, but could as well be due to hemispherical clamping once again. Similarly, it would be interesting to measure correlations across different color channels for a same moment order.

In this work, we have concentrated on measured BRDF data, since they are closest to real-world materials. However, they may also be prone to measurement errors; in particular, data at highly grazing angles tend to be less reliable. In future work, we plan to study analytical BRDF models with a similar statistical analysis and compare results to the present analysis. The advantages of working with analytical models is that components are readily separated, and arbitrary grazing angles may be studied. We also plan to explicitly take surface curvature and viewing projection into account in our local Fourier analysis. This will permit to directly establish relationships between image and material properties in complex 3D scenes. In the long term, we are interested in extending our study to full 4D (anisotropic) BRDFs. However, this will likely require to find a more appropriate parametrization for BRDF slices. Finally, we believe that BRDF moments may also be well adapted to the study

of material perception. In this regard, we are interested in conducting perceptual experiments in the vein of the work of Wills et al., ${ }^{4}$ with a focus on statistical moments. The end goal of such an approach is to explicitly connect perceptually-relevant gloss properties to measurable BRDF properties.

\section{REFERENCES}

[1] Nicodemus, F. E., "Directional reflectance and emissivity of an opaque surface," Appl. Opt. 4, 767-775 (Jul 1965).

[2] Kajiya, J. T., "The rendering equation," SIGGRAPH Comput. Graph. 20, 143-150 (Aug. 1986).

[3] Hunter, R. S., [The measurement of appearance], John Wiley \& Sons (1987).

[4] Wills, J., Agarwal, S., Kriegman, D., and Belongie, S., "Toward a perceptual space for gloss," ACM Trans. Graph. 28, 103:1-103:15 (Sept. 2009).

[5] Ngan, A., Durand, F., and Matusik, W., "Experimental analysis of brdf models," in [Proceedings of the Eurographics Symposium on Rendering], 117-226, Eurographics Association (2005).

[6] Matusik, W., Pfister, H., Brand, M., and McMillan, L., "A data-driven reflectance model," ACM Transactions on Graphics 22(3), 759-769 (2003).

[7] Bulmer, G., [Principles of Statistics], Dover Books on Mathematics Series, Dover Publications (1979). 
[8] Ghosh, A., Chen, T., Peers, P., Wilson, C. A., and Debevec, P., "Estimating specular roughness and anisotropy from second order spherical gradient illumination," in [Computer Graphics Forum], 28, 4 (June 2009).

[9] Havran, V. and Sbert, M., "Statistical Characterization of Surface Reflectance," in [Eurographics Workshop on Material Appearance Modeling], Klein, R. and Rushmeier, H., eds., The Eurographics Association (2014).

[10] Ramamoorthi, R. and Hanrahan, P., "A signal-processing framework for reflection," ACM Transactions on Graphics 23(4) (2004).

[11] Durand, F., Holzschuch, N., Soler, C., Chan, E., and Sillion, F. X., "A frequency analysis of light transport," ACM Transactions on Graphics 24(3), 1115-1126 (2005).

[12] Belcour, L., Soler, C., Subr, K., Holzschuch, N., and Durand, F., "5D Covariance tracing for efficient defocus and motion blur," ACM Transactions on Graphics 32(3), 31:1-31:18 (2013).

[13] Ramamoorthi, R., Mahajan, D., and Belhumeur, P., "A first-order analysis of lighting, shading, and shadows," ACM Transactions on Graphics 26(1) (2007).

[14] Phong, B. T., "Illumination for computer generated pictures," Commun. ACM 18, 311-317 (June 1975).

[15] Burley, B., "BRDF Explorer." http://www.disneyanimation.com/technology/brdf.html (2012).

[16] Koenderink, J. and Pont, S., "The secret of velvety skin," Mach. Vision Appl. 14, 260-268 (Sept. 2003).

[17] Vangorp, P., Laurijssen, J., and Dutré, P., "The influence of shape on the perception of material reflectance," ACM Trans. Graph. 26 (July 2007).

- yellow-phenolic

- yellow-matte-plastic

- white-paint

- white-marble

- white-acrylic

- violet-acrylic

- two-layer-gold

- tungsten-carbide

- $\operatorname{ss} 440$

- specular-violet-phenolic

- specular-green-phenolic

- specular-blue-phenolic

- specular-black-phenolic

- silver-paint

\section{LIST OF UNIMODAL MATERALS}

- silicon-nitrade

- red-metallic-paint

- pvc

- pure-rubber

- pearl-paint

- nickel

- neoprene-rubber

- hematite

- green-metallic-paint2

- green-metallic-paint

- gold-paint

- gold-metallic-paint3

- gold-metallic-paint2

- color-changing-paint3
- color-changing-paint2

- color-changing-paint1

- chrome

- chrome-steel

- brass

- blue-metallic-paint2

- blue-metallic-paint

- black-phenolic

- black-obsidian

- aventurnine

- aluminium

- alum-bronze

\section{VARIANCE OF LAMBERTIAN}

A Lambertian material corresponds to $\rho_{L}=\frac{1}{\pi^{2}}$ in our angular parametrization, irrespective of the view direction. Since we are working in a closed space $\left[-\frac{\pi}{2} \cdot . \frac{\pi}{2}\right]^{2}$, the formulation of moments for a constant function is not the same than in an infinite domain: moments of a Lambertian BRDF become finite. We can simplify the expression of the co-variance matrix since the mean of a Lambertian BRDF slice is always zero: $\mu_{1,0}\left[\bar{\rho}_{L}\right]=\mu_{0,1}\left[\bar{\rho}_{L}\right]=0$, hence $\Sigma_{n, m}\left[\bar{\rho}_{L}\right]=E_{\bar{\rho}_{L}}\left[\theta_{i}^{n} \phi_{i}^{m}\right]$ for $n+m=2$. Furthermore, variances along $\theta_{i}$ and $\phi_{i}$ will yield the same result due to the symmetry of the integration space and integrands. Thus the average variance is equivalent to the variance along $\theta_{i}$, leading to the formula:

$$
\sigma^{2}\left[\rho_{L}\right]=\frac{1}{\pi^{2}} \iint_{\theta_{i}, \phi_{i} \in\left[-\frac{\pi}{2} \cdot \frac{\pi}{2}\right]^{2}} \theta_{i}^{2} d \theta_{i} d \phi_{i}=\frac{1}{\pi}\left[\frac{\theta_{i}^{3}}{3}\right]_{-\frac{\pi}{2}}^{\frac{\pi}{2}}=\frac{\pi^{2}}{12} .
$$

